\title{
Bioanalysis
}

\section{EBF recommendation for stability testing of anti-drug antibodies; lessons learned from anti-vaccine antibody stability studies}

Long- and short-term stability testing of the analyte is one of the key parameters in bioanalytical method validation in support of pharmacokinetics. However, for immunogenicity testing, the scientific rationale for long- and short-term stability testing on quality control samples most often spiked with polyclonal antibody raised in a different species should be questioned. Therefore, the European Bioanalysis Forum (EBF) formed a Topic Team to discuss the scientific rationale for stability testing of anti-drug antibodies (ADAs). A review of EBF member companies' experience on ADA stability and on anti-vaccine antibodies from vaccine projects was the basis of this discussion. EBF recommends to perform short-term stability testing of the positive control, but not to perform long-term stability testing of ADAs in nonclinical and clinical studies.

\section{Background \& scope}

Testing for unwanted immunogenicity towards biological drugs is part of the analytical program for nonclinical and clinical studies. Anti-drug antibody (ADA) responses are frequently elicited in nonclinical studies as the protein or peptide drug is often of human origin (e.g., a fully human or humanized monoclonal antibody). The evaluation of the ADA response is mainly used to explain impacted pharmacokinetic profiles and toxicity findings. Owing to the human nature of the drug and the limitation in animal models, it is generally accepted that the ADA response in nonclinical species is not predictive for the human ADA response. In humans, the impact of ADAs can be of safety and efficacy concern; therefore, it is important to investigate the ADA response. Recommendations in the present paper apply to both nonclinical and clinical studies.

Validation procedures and acceptance criteria for stability testing should be based on international guidelines, internationally recognized conference reports, scientific publications $[1-8]$ and scientific rationale. According to the US FDA draft immunogenicity guidance "Patient samples should be stored in a manner that preserves antibody reactivity at the time of testing". It is further stated, that "Freezing and thawing patient samples may also affect assay results and those results should be evaluated" [3]. However, no detailed recommendations for ADA stability testing is specified in the current guidelines from the EMA and FDA [3-7] and specific papers for immunogenicity testing $[1,2,8]$. These guidances are nevertheless used by many companies as the basis for the set-up of stability testing of ADAs.

Long-term stability testing using spiked quality control (QC) samples reflect the stability of 'artificial' positive controls stored under certain conditions over time. It is commonly admitted that the positive control is unlikely to reflect the real study samples. Shankar et al. stated, "It is reasonable to assume that the stability of ADA is the same whether it is specific to drug $X$ or drug Y. With this logic, the stability of ADA can be approximated by the stability of serum or plasma immunoglobulin specific to any antigen" [2]. Having this in mind, the link to vaccine programs is of great value as stability testing has here been performed to a much greater extent. The reason for this is
Susanne Pihl*,1, Lydia Michaut ${ }^{2}$, Jenny Hendriks ${ }^{3}$, Ralf Loebbert ${ }^{4}$, Janka Ryding ${ }^{5}$, Martin Nemansky 6 , Laurent Vermet ${ }^{7}$ \& Arjen Companjen ${ }^{3}$

${ }^{1} \mathrm{H}$ Lundbeck A/S, Department of Bioanalysis, Drug Development ADME, H Lundbeck A/S, Ottiliavej 7-9, DK-2500 Valby, Denmark

${ }^{2}$ Novartis Institutes for Biomedical Research, DMPK-Biologics, Bioanalytics, Klybeckstrasse 141, CH-4057 Basel, Switzerland

${ }^{3}$ Crucell Holland BV, Archimedesweg 4-6, PO Box 2048, 2301 CA Leiden, The Netherlands

${ }^{4}$ AbbVie Deutschland GmbH \& Co. KG; DMPK-BA, Development Sciences; Knollstrasse, Ludwigshafen, Germany ${ }^{5}$ Ferring Pharmaceuticals A/S, Bioanalysis, International Pharma Science Center, Kay Fiskers Plads 11, DK-2300 Copenhagen S, Denmark

${ }^{6}$ PRA, Bioanalytical Laboratory, Westerbrink 3, 9405 BJ Assen,

The Netherlands

${ }^{7}$ Sanofi Research \& Development - Drug Safety Animal Research - Biomarkers \& Biological assays, Montpellier, France *Author for correspondence: supi@lundbeck.com 
that, in contrast to studies for biological drugs, vaccine studies pursue the antibody response against the vaccine, hence resulting in much more samples containing acquired antibodies. This enables a thorough study on antibody stability, typically in serum.

Here, the European Bioanalysis Forum (EBF) reports back from their internal discussions on stability testing of ADAs performed in the regulated bioanalytical environment. These discussions are an integral part of an EBF Topic Team assigned to provide a recommendation on the scientific rationale for stability testing of ADAs. The discussion started at a break-out session on large molecule stability at the EBF Open Symposium in Barcelona in November 2011, and resulted in the initiation of the Topic Team 'Stability of ADAs' at EBF's Strategy Meeting in March 2012. The discussions started questioning the relevance of performing long-term stability testing on QC samples spiked with a surrogate positive control, most often a polyclonal antibody raised in a different species. The discussion focused on the need for stability testing in general for ADAs. Data on stability testing of antivaccine antibodies (AVAs) can serve here as a relevant model. A survey was therefore carried out within the EBF community to assess the current practices and any relevant results for ADA stability testing. The survey did not distinguish between nonclinical and clinical stability testing.

\section{Current recommendation \& general practice for stability testing}

In many companies, ADA stability testing is derived from best practices in regulated, quantitative bioanalysis, which include long- and short-term stability testing. Short-term stability testing of the analyte commonly includes bench-top, freeze/thaw and processed sample stability. Long-term stability testing should justify that no significant degradation of the analyte will occur at the required storage period and condition, starting at the time of sample collection to the time of analysis [6,7]. These experiments are intended to reflect the expected sample handling and storage conditions in order to ensure the integrity of the analytical data (Box 1).

\section{Key terms}

ADAs: Anti-drug antibodies induced by biological therapeutic molecules.

Stability testing: Stability tests of samples in the chosen sample matrix, container and storage conditions.

Long-term stability: Stability of samples when stored for a duration longer than the validation.

AVAs: Anti-vaccine antibodies induced upon vaccination with either a preventive or a therapeutic vaccine.
Long-term stability should cover the period from the time a sample is taken to the last analysis, and should be tested in the relevant container system and matrix after storage at the relevant period and temperature $\left(-20^{\circ} \mathrm{C}\right.$ or $\left.-80^{\circ} \mathrm{C}\right)$.

Short-term stability testing consists of bench-top stability and freeze/thaw stability. It is stated by MireSluis et al. [1] that it is difficult to predict sample stability with a surrogate positive control. It is recommended to reduce the number of freeze/thaw cycles by storing aliquots of the samples. The US Pharmacopeial Convention guidance states that "It is generally accepted that a human ADA sample in serum or plasma will be stable after three freeze/thaw cycles" [8]. Nevertheless, freeze/thaw stability should be evaluated. Investigation of the stability of the final processed samples should be considered based on the method and storage conditions.

In total, $27 \mathrm{EBF}$ companies dealing with biological drug development participated in the survey that was initiated to investigate the best practices in the stability testing of ADAs and AVAs. A total of $70 \%$ (19/27) of the survey's responding companies routinely assess short-term ADA stability. A minority of $5 \%(1 / 19)$ do not assess freeze/thaw and bench-top stability, whereas $59 \%(16 / 27)$ of the survey responders routinely perform long-term ADA stability testing. All responders use spiked QC samples for stability testing. Seven member companies were able to share data on long-term stability encompassing ADAs against more than 48 different biological drugs including peptides, proteins and monoclonal antibodies and AVAs against one vaccine. No instability was observed for the investigated ADAs and AVAs.

In a second survey, additional questions were asked to obtain the experience on ADA and AVA long-term stability testing to include the EBF companies, which were not able to share data. Data from companies able to share data was excluded in the summary of the second survey to avoid an overlap with the first survey. None of the companies that replied in the second survey experienced any long-term instability.

A summary of the surveys regarding long-term stability testing was shown in Table 1.

\section{Acceptance criteria for stability testing of ADAs}

Neither guidelines, nor relevant scientific papers provide clear guidance for the set up, as well as the acceptance criteria of the stability testing for ADAs [1-8]. It is therefore important that acceptance criteria for stability testing are predefined in a standard operating procedure or study plan. The practical setting of acceptance criteria for ADA stability strongly depends 


\section{Box 1. Definition of short- and long-term stability as per the US FDA guidelines.}

\section{Short-term stability}

- Bench-top stability is assessed by keeping stability samples at room temperature for $4-24 \mathrm{~h}$, the duration depending on the intended study

- Freeze/thaw stability is assessed for the number of anticipated freeze/thaw cycles during analysis. Samples are stored frozen for a minimum of $24 \mathrm{~h}$ and then thawed unassisted at room temperature. When thawed, the samples are refrozen for 12-24 h

- Stability of processed samples is the test of the final processed sample prior to detection

Long-term stability

- Long-term storage stability studies should be designed to confirm analyte stability in the test system matrix and container covering the length of time from sample collection to sample analysis

Data taken from [7].

on the methodological approaches (e.g., signal values or titer results as read out). In addition, different analytical levels are applied for stability testing of the ADAs, such as scoring (negative/positive), a titer value and/or signal responses.

Results of the EBF survey revealed that $27 \%$ of the 22 responding companies testing stability based their stability acceptance criteria on the fact that a sample consistently yields a positive assay response.

A different approach is to include the titer result in the acceptance criteria for stability testing. For $18 \%$ of the responders, the stability is accepted if the titer level is within the acceptance criteria range (i.e., consistent with a level determined during method validation).

Half of the responders (50\%) apply the following acceptance criteria: a certain amount of stability samples have to return a response within a signal limit, for example, $\pm 20 \%$ of the mean/original signal variations of this approach include the direct signal comparison of stability of QC samples with 'freshly' prepared QC samples that should not deviate more than a predefined value (e.g., 20 or $25 \%$ ). Another approach mentioned in the survey takes into account the ratios of the QC (e.g., low QC/negative control and high $\mathrm{QC} /$ low $\mathrm{QC}$ ) that need to be within the predefined acceptance criteria ranges.

The outcomes of the different stability testing acceptance criteria do not reveal remarkable differences nor lead to contradicting conclusions. This EBF paper aims at summarizing the common practices rather than recommending or promoting one way over another. The applied approaches should be based on sound scientific rationale and acceptance criteria should be predefined in a standard operating procedure or study plan.

\section{ADA \& AVA responses are similar}

Since vaccines work by eliciting an immune response that will protect the body from an invading pathogen or a deleterious version of a self-protein, determining the immunogenicity of a vaccine in development is the basis for discovery in nonclinical and clinical development. The purpose of immunogenicity assays for vaccines is to predict whether a vaccinee will be protected against infection or disease caused by the pathogen or molecule that is targeted by the vaccine. Since with vaccination, a possible correlation of protection requires an accurate and precise determination of the protective antibody response level, a validation of the assay is required.

Currently, no validation guidelines for vaccine immunogenicity exist [9], therefore, the FDA and EMA guidelines for bioanalytical method validation $[6,7]$ are adopted and adjusted for ligand binding or cellbased assays where necessary. Vaccine assays are generally designed for quantitative determination [9]. As per FDA and EMA guidances on bioanalytical method validation, assessment of analyte stability is required as part of assay validation, taking into account that a true reference of the analyte is available. For AVA studies, in many cases, a serum pool from pathogen-exposed individuals is available, mimicking much more closely the samples that would be generated upon vaccine administration as a 'true, reference.

Table 1. Results of the surveys regarding long-term stability testing.

\begin{tabular}{|llll|}
\hline Survey & $\begin{array}{l}\text { Companies providing data on testing } \\
\text { ADA/AVA long-term stability }(\mathrm{n})\end{array}$ & $\begin{array}{l}\text { ADA/AVA assessments in long-term stability } \\
\text { studies }(\mathrm{n})\end{array}$ & $\begin{array}{l}\text { Any instability } \\
\text { detected }\end{array}$ \\
\hline 1 & 7 & $>48$ (ADA); 1 (AVA) & No \\
\hline 2 & 3 & 7 (ADA); 1 (AVA) & No \\
\hline Total & 10 & $>55$ (ADA); 2 (AVA) & No \\
\hline ADA: Anti-drug antibody; AVA: Anti-vaccine antibody. & & \\
\hline
\end{tabular}


In principle, antibodies to a biological drug are the same in nature; it is the intended purpose of the biological drug (vaccine or drug) that determines whether the response is wanted or unwanted. It could be argued that based on this principle, results of AVA stability testing, could be directly extrapolated to stability testing of ADAs.

In this same issue of Bioanalysis, Michaut et al. [10] demonstrates the stability of anti-vaccine $\operatorname{IgG}$ that was stored for up to 3.6 years at $-80^{\circ} \mathrm{C}$. The mean percentage difference between the titers initially determined and those re-assessed after 2.1-3.6 years in 62 serum samples from 32 different donors was $-3 \%$ with a median of $-5.6 \%$ (range: $-25.7-24.5 \%$ ). No significant variability was observed between individual donors nor between titer levels, showing that, in general, anti-vaccine IgGs are stable in human serum for at least 3.6 years after collection and stored at a nominal temperature of $-80^{\circ} \mathrm{C}$ in a temperature-monitored freezer.

In addition, in this same issue of Bioanalysis, Hendriks et al. [11] also confirms stability of anti-vaccine polyclonal antibodies for up to 4.6 years at $-80^{\circ} \mathrm{C}$, both for IgG-binding antibodies and for functional neutralizing antibodies. Stability of polyclonal antibody response was also shown for samples shipped on dry ice, showing that no loss of activity is introduced during shipping. It should be kept in mind that this is equivalent to storage at $-80^{\circ} \mathrm{C}$ for a short time. Even when introducing up to eight freeze/thaw cycles, the antibody-binding capacity remains within acceptable ranges, although a trend in decrease of $13 \%$ in titer can be observed [11].

\section{Conclusion \& recommendation for stability testing of ADAs}

This paper provides data from an EBF company member survey demonstrating that instability was not seen for ADAs against more than 55 different biological drugs, for which long-term stability was tested. These data are supported by references to long-term stability studies conducted with AVAs, which successfully demonstrate stability in all investigated cases. The antibody response being the same in nature for both ADAs and AVAs, it is therefore concluded that it is not relevant to assess long-term stability of ADAs. This confirms

\section{References}

1 Mire-Sluis AR, Barret YC, Koren E et al. Recommendations for the design and optimization of immunoassays used in the detection of host antibodies against biotechnology products. J. Immunol. Methods 289, 1-16 (2004).

2 Shankar G, Devanarayan V, Amaravadi L et al. Recommendation for the validation of immunoassay used for
Shankar et al.'s postulate that stability of ADAs is the same independent of the biological drug [2]. This is also in alignment with the US Pharmacopeial Convention Guidance [8], which states that it is generally accepted that samples stored at $-70^{\circ} \mathrm{C}$ are stable and should therefore not be tested.

However, bench-top storage of the samples at higher temperatures $\left(4^{\circ} \mathrm{C}\right.$ or ambient), as well as freezing and thawing them represent more stressful conditions for the samples. Therefore, it is considered relevant to include short-term stability testing in the method validation.

The recommendation in summary:

- The EBF recommends that long-term stability testing of ADAs is not necessary to validate clinical and nonclinical study results;

- The EBF recommends assessing short-term stability (freeze/thaw and bench-top stability experiments) of ADAs as part of the assay validation;

- Depending on the method used and storage condition of the finally processed sample, the relevance of the investigation of the processed sample stability should be considered.

\section{Acknowledgements}

The authors would like to thank all European Bioanalysis Forum IGM members that contributed to the surveys.

\section{Disclaimer}

The views and conclusion presented in this paper are those of the European Bioanalysis Forum and do not necessarily reflect the representative affiliation or company's position on the subject.

Financial \& competing interests disclosure

The authors have no relevant affiliations or financial involvement with any organization or entity with a financial interest in or financial conflict with the subject matter or materials discussed in the manuscript. This includes employment, consultancies, honoraria, stock ownership or options, expert testimony, grants or patens received or pending, or royalties.

No writing assistance was utilized in the production of this manuscript.

detection of host antibodies against bioanalytical products. J. Pharm. Biomed. Anal. 48, 1267-1281 (2008).

3 US Department of Health and Human Services, US FDA, Center for Drug Evaluation and Research, Center for Biologics Evaluation and Research. Draft Guidance for Industry - Assay Development for Immunogenicity Testing of Therapeutic Proteins. US FDA, MD, USA (2009). 
4 European Medicines Agency, Committee for Medical Products for Human Use. Guideline on Immunogenicity Assessment of Biotechnology-Derived Therapeutic Proteins. European Medicines Agency, London, UK (2006).

5 European Medicines Agency, Committee for Medical Products for Human Use. Guideline on Immunogenicity Assessment of Monoclonal Antibodies Intended for In Vivo Clinical Use. European Medicines Agency London, UK (2010).

6 European Medicines Agency, Committee for Medical Products for Human Use. Guideline on Bioanalytical Method Validation. European Medicines Agency, London, UK (2011).

7 US Department of Health and Human Services, US FDA, Center for Drug Evaluation and Research, Center for
Veterinary Medicine. Guidance for Industry, Bioanalytical Method Validation. US FDA, MD, USA (2001).

8 USP General Chapter. Immunogenicity assays - design and validation of immunoassays to detect anti-drug antibodies. Pharmacopeial Forum (PF) 38(3), 1106 (2012).

9 Kostense S, Hendriks J, Challenges of immunogenicity assays for vaccines. Bioanalysis 2012 4(4), 397-406 (2012).

10 Michaut L, Laurent N, Kentsch K, Spindeldreher S, DeckertSalva F. Stability of anti-immunotherapeutic antibodies infrozen human serum. Bioanalysis 6(10), 1395-1407 (2014).

11 Hendriks J, Stals C, Versteilen A et al. Stability studies of binding and functional anti-vaccine antibodies. Bioanalysis 6(10), 1385-1393 (2014). 